\title{
XIX. Memoir on the saccharine diabetes
}

\section{Messr. Dupuytren \& Messr. Thenard}

To cite this article: Messr. Dupuytren \& Messr. Thenard (1806) XIX. Memoir on the saccharine diabetes, Philosophical Magazine Series 1, 26:102, 97-105, DOI: 10.1080/14786440608563643

To link to this article: http://dx.doi.org/10.1080/14786440608563643

$$
\text { 册 Published online: } 18 \text { May } 2009 .
$$

Submit your article to this journal $\pi$

LII Article views: 2

Q View related articles $\asymp$ 
XIX. Meinoir on the Saccharine Diabetes. By Messrs. Dupuytren und Thenard *.

$I_{r}$ has been long known that the human urine is so strangely altered in the disease called dialietes, that in place of being in a small quantity and acrid, like that of a person in health; it becomes, on the contrary, saccharine and very abundant. It is only about thirty years, however, since the first analyses were made of diabetic urine. Three cduses retarded this investigation: on the one hand, the infrequency of the disease; on the other, the uncertainty of the chemical apparatus formerly employed; and, thirdly, the neglect which animal chemistry has suffered until within these very few years.

It was not sooner than 1778 that the existence of sugat in diabetic urine was demonstrated. This discovery, for which we are indebted to Caulcy, was established in $179 \mathrm{i}$ by Franck. It was, indeed, hinted at by Willis, at the beginning of the 17 th century; and afterwards, in 1775 , by Pool and Dobson. M. Caulcy, however, having only directed his attention to the sacchirine substance alone; left a great deal undone. It was necessary to ascertain the nther principles of diabetic urine, and particularly those which enter into common urine; and this was accomplished in 1803 by Messrs. Nicholas and Guendeville of Caen. It results from their researches that the urine of diabetic patients does not contain a sensible quantity of urea, or of uric acid; that the most sensible reagents scarcely indicate traces of phosphate and sulphate; that it is impossible to discover a free acid in it; and, in short, that nothing is found except sugar in great quantity, and more or less marine salt.

In the present memoir we purpose not only to confirm the results we have cited, but in particular we shall communicate,

1st, The medical observations made by us upor the diabetic patient whose urine we analysed.

* From Ammles te Chimie, tom. lix. p. 41.

Vol; 26. No. 102. Nov: 1806. G 
2d, The very peculiar nature of the saccharine substance we found in that urine.

3d, The different transformations which it underwent before being bruught back to its natural appearance and consistency.

\section{PART FIRST.}

Medical Observations upon the Case in which we analysed the Diabetic Urine.

It resulted from these observations :

Ist, That the saccharine diabetes may last several yeary; and even as long as the digestive faculties continue, and are able to administer to the excessive losses which take place through the urine.

$2 d$, That this disease is not incurable in any of its stages, not even when an altered digestion seems no longer to furnish matters of secretion and the animal ceconomy is exhausted.

3d, That the stat of this affection seems to be in the kidneys and not in the intestinal canal.

In fact, the appetite and thirst of diabetic patients are not perverted; they appear merely to be in proportion to the want of organical repair, as well as the digestive faculties; in the second place, the alimentary substances undergo the same operation in the stomach of a diabetic patient as in that of a healthy person; and what proves that the digestion is not altered, but merely increased, in diabetes is, the quantity of food taken, the rapidity with which it is digested, the great proportion of matter which is absorbed from that food, and the small quantity of freces to which it is reduced : in short, we find no saccharine liquid, or any liquid which has undergone any alteration in its composition, all the way from the organs of digestion to those of the urinary secretion.

4th, That the cause of saccharine diabetes appears to bo 2 heightened and perverted action of the kidneys.

That in consequence of this action the saccharine matter of urine is produced, and to this cause we may ascribe all the symptoms of this malady. 
3th, That the excessive losses which take place in this disease seem to ascertain, in some circumstances, a very considerable absorption at the surface of the body in diabetes.

6th, That the new rehations determined by sacharine diabetes between the aliments and the secretions in general, and between each of their specres in particular, are arialogous to those which are determined by an excessive evacuation, of whatever kind it may be.

7th, That the treatrient prescribed by Rollo, and afterwards èmployed with so much success by our countrymen Messrs. Nicholàs and Guendeville, and which consists in a regimen purely animal, bas the same degree of efficacy in diabetes as Jesuits' bark has in intermittent fevers.

8 th, Finally, that the saccharine diabetes produces no other change in the state of our organs than a development of the digestive and urinary apparatus; both of which are highly active during this disease, the one in preparing and the other in discharging the alimentary substances.

\section{Part Second.}

Analysis of the Urine evaruated ly a Diabetic Patient, from the 15th Day of his Admission into the Hotel-Dieu until he was carried into the Infirmary of the School of Mcdicine of Paris.

The above urine came from the patient in an uncommonly large quaritity, and exhaled a smell by no means disagreeable.

It was limpid, yellowish, specifically heaviet than water, and scarcely reddened the tinctire of turnsole; slightly sact charine, it had at the same time something of the taste of sea salt.

Left to itself at a temperature of $15^{\circ}\left(59^{\circ} \mathrm{Fahr}\right.$. $)$, it became turbid in five or six days; bubbles of carbonic acid were liberated from it if it was ever so little shaken; the urinous smell it had at first was dispelled; and it contracted a smell analogous to that of newly made wine: it yielded alcohal upon being distilled, and becane stroly acidified on exposure G 2 
to the air; it therefore presented, in a weak degree, all the characters of a spirituous fermentation.

When distilled in a retort, or evaporated in a capsule, the phanomena were the same; it became turbid, thickened by little and little, and was reduced to a syrup sometimes equivalent to a seventeenth, sometimes a twentieth, but never less than a thirticth of its own weight. We extracted in this manner from the urine we treated, nearly thirty pounds of this syrup, which upon cooling always became one mass, composed of a multitude of small grains without consistency. These soft granulated crystals being hardly sweet, it was natural to think that the substance of which they were formed was not homogeneous, and contained only a very small quantity of the saccharine principle. In order to ascertain it, the following experiments were made:

We took 100 parts of this substance and distilled it in a retort, the neck of which was inserted info a receiver which was continually kept at a low temperature. We obtained plenty of water, a little oil, and no ammonia ; a great quantity of gases a little fetid, and abundance of charcoal easily incinerated, yielding, upon complete incineration, two parts and ahalf of sea salt and one half part of phosphate of lime.

From this result may be drawn the three following consequences:-1st, That this substance contains no auimal matter, since when calcined it yields no rolatile alkali : 2d, That it contains very little saline matter, because when reduced to ashes it only presents a residue equal to some one hundredth parts of its weight: $3 \mathrm{~d}$, That it is formed of vegetable principles alone, because it gives all the products of vegetables upon being distilled.

Presuming that the sugar was one of these principles, and forming no kind of conjecture upon the nature of those with which it was supposed to be mixed, we resolved to employ fermentation to destroy the former, and to keep the latter principles unaltered, in such a manner, that by filtration and evaporation we ought to have obtained them very pure. We mixed in a large flask 100 grammes of the substance to be analysed, 25 grammes of ferment, and $\mathbf{9 0 0}$ 
grammes of water; we adapted to the neck of this flask a tube fixed under a flask full of water; the temperature being then raised to 18 degrees, the experiment was left to itself. Some hours after the contact took place between these substances a motion was evinced in some parts of the liquor, which soon became general : plenty of solid flakes, which gave birth to a great number of gaseous bubbles, were raised to a considerable height; these bubbles rapidly passed into the vessels full of water, but the flakes fell back to the bottom of the flask, and, giving birth to new bubbles, they again ascended to be onçe more precipitated. This phænomenon, which took place for three days, announced a very active fermentation, and, consequently, the presence of a great quantity of saccharine matter: in fact, more than thirteen pints of pure carbonic acid gas were liberated; the liquor was very alcoholic, and contained nearly 48 parts of alcohol at 40 degrees; evaporated to dryness, only 23 parts of extract were obtained, formed of three parts of sea salt and twenty parts of a viscous brown matter.

We know that 100 grammes of sugar produce 12 grammes of a similar zesidue, 56 of alcohol, and 36 of carbonic acid. Thus the substance drawn from diabetic urine yielded by fermentation the same products, and almost in as great quantity, as pure and finely crystallized sugar; and if we add, that it acts like sugar with the nitric acid, alcohol, and the other reagents, we must regard these two substances as being in some measure precisely the same.

We ought, however, to recollect that it is scarcely sweet, at least much less so than sugar. It may be thence con* cluded, 1st, That, as has been long suspected, there are different species or varieties of sugar, the differences being so striking as now to render certain what before was only probable. But as the taste only is not a certain criterion of the saccharine principle, it becomes necessary to examine if, among the substances which have hitherto been confounded with sugar on account of their taste, there are not some of them which differ essentially from that substance.

For this reason we were led to examine manna. Our first care was to mix it with ferment and water at the tempera- 
ture of $18^{\circ}\left(64^{\circ} \mathrm{Fabr}.\right)$, and to remark attentively all the phænomena produced by this mixture. Fermentation soon began : it was brisk at first, but it soon decreased; and in two days it ended. The liquor had nevertheless a very strong vinous smell; but far from being alcoholic, it was, on the contrary, very saccharine, and deposited upon evaporation, in the form of crystals, almost all the matter enployed, deprived of the fermenting faeulty.

Although convinced by these results that manna contained only a very small quantity of sugar, we ought nevertheless to compare it in all its properties with this substance, in order to place the fact beyond a doubt, and thus to discover all the characters peculiar to the particular principle of which it appeared almost entirely formed: we therefore tried its action on spirit of wine, which did not attack the saccharine principle, and upon the nitric acid, which did not convert any portion of that principle into mucous acid.

The former of these reagents, at the temperature of $60^{\circ}$ $\left(140^{\circ} \mathrm{Fahr}.\right)$, dissolved so great a quantity of manna, that, upon cooling, the liquor was a mass composed of a crowd of crystalline heaps, every crystal in each heap springing from a common centre. The second reagent produced, afier a long ebullition, so great a deposit of mucous acid, that it was almost half the weight of the manna employed.

Here, therefore, there are two characters which are strikingly different; sugar properly so called, and the particular principle of manna,

New researches will doubtless present many other differences more or less prominent; but those above related being sufficient to oocasion these bodies to be regarded as very distinct from each other, we did not think it necessary to enter more deeply into the subject.

It follows from bence, that it would be always easy to recognize and separate manna, or rather the peculiar principle of manna, whatever may be the substances with which it is mixed. It is only necessary to treat with warm alcobol the matter which contains this peculiar principle of manna, and it will be almost entirely precipitated upon cooling, In truth, there are other vegetable substances which possess 
this principle even in a remarkable degree; but these substances always hold some acid in their composition, from which they may be freed by combining it with an alkaline or earthy base, or a metaltic oxide, according to the nature of the acid; and consequently this made of separation may be generally employed.

It is in this manner that we may ascertain if the honeylike substance observed upon the leaves of certain trees, and particularly on those of the linden tree, is in reality a kind of manna; and if the saccharine principle which exists in asparagus is of the sante description; it having been lately discovered by Messrs. Vauquelin and Robiquet, that in asparagus the saccharine substance is mixed with a principle entirely peculiar*.

\section{Analysis of the Urine etacuated by the Patient from the}

Time he was brought to the Infirmary of the School of Medicine until he went out cured.

Dusing the whole time the patient was in the Hotel-Dieu he could not be subjected to any particular regimen. He lived as he pleased; his disease therefore remained stationary, and his urine, at all times very abundant, was never changed in its nature, It was then resolved to carry him to the Infirmary of the School of Medicine, where, being almost continually watched by M. Dupuytren and his pupils, it became easier to make him do whatever he was desired.

After a few days all kinds of vegetables were refused, and animal food only was given him. The weights of what he ate, and of the liquids he drank to quench a most dreadful thirst, were ascertained.

For the first two or three days no change was remarked in his urine; hut in five or six days it became less white, sharper, more acid, and less saccharine; when submitted to evaporation, in place of continuing limpid as before, it became turbid, and was covered with a thick pellicle of albuminous matter. When I perceived this change, particularly the presence of an animal matter in his urine, although the

* The paper here referred to will be given in a subsequent number.

G 4 
state of the patient was absolutely unknown to me, and I knew nothing of the treatment which he had undergone, I presumed that the disease'began to yield: having afterwards observed this animal matter to become daily more abundant, I regarded the cure of the patient as at no great distance : I communicated my opinion to M. Dupuytren, who seemed surprised at my prediction; this surprise, however, ceased when I informed him what had taken place in the patient's urine.

From this period the patient became better and better. Fis urine was daily more animalized and less saccharine. The antimal albuminous substance gradually diminished also, and the ure and uric acid began to appear. His urine then became perfectly similar to that of a person in health. In other words, he was cured; but, having given himself up to various exccsses, he soon fell ill again ; the diabetes again made its appearance, accompanied with other diseases which had also formerly attended it in the same patient.

If we resume, however, all the consequences which may be drawn from the experiments we have related in the second and third parts of this memoir; we may assert,

1st, That the urine of the diabetic patient above examined was almost entirely çomposed of a matter only a little sacharine; that nevertheless this matter enjoys all the properties which eharacterize sugar; because it is transformed into alcohol and carbonic acid by fermentation; it yields plenty of oxalic acid and no mucaus acid by the nitric acid; it is very little soluble in alcohol at $36^{\circ}$; it produces when calcined little oil, and plenty of water and carbonic acid; thus it is clearly demonstrated that there are different varieties of sugar.

$2 \mathrm{~d}$, That manna is not a species of sugar; that it only contains small quantity, which may be destroyed by fermentation; that it contains, on the contrary, plenty of a peculiar prineiple, the taste of which is very sweet, and the character of it is not to ferment with yeast; it gives plenty of mucous acid with the nitric acid, is more soluble in warm than in cold water; but, above all, it is soluble in alcohol to such a degree, that, upon cooling, the solution becomes a crystalline mass, 
3d, That upon giving nothing but animal food to diabetic patients, their urine speedily changes; that at first we find an albuminous matter in it; that this albuminous matter, which always increases for some days, is an unequivocal sign of a speedy cure ; that afterwards this albumen gradually disappears; that the kidneys then begin to secrete the substance called urea, uric acid, and also the acetous acid; the urine soon becomes like that of an individual in health.

Notwithstanding all this, however, the patient, in onder to prevent a relapse, ought to observe the animal regimen for a long time, and avoid every thing which may tend to cause the reappearance of diabetes.

XX. Memoir upon Animal Fat, and some Medicinal Preparations which are administered through that Medium, By $M$, Voget.

[Concluded from p. 81.]

\section{Fat and the Metals.}

$I_{T}$ has been ascertained that fat acts more or less upon a great number of the metals. Copper, for instance, gives a green colour to fat, when the air also is allowed to act upon it. The solution of the oxide of copper in fat is a fact proved by daily experience.

As mercury, however, is the metal which excites most interest in the pharmaceutic art in its combinations with fat, I shall restrict myself particularly to discussing the effects of these combinations.

Several apothecaries have endeavoured to improve the quality of mercurial ointments, and particularly of the double ointment. M. Vean-Delaunay proposed rancid oil, and M. Fourcroy has shown that oxygenated fat is most proper for killing mercury.

Of late, several chemists have imagined that the mercury of the double mercurial ointment is not oxidated, but that it exists in the metallic state. 